$\star$ Symposium on Intravascular Catheterization (Chairman. Prof. Dr. J. MISE)

\title{
Studies on Right Ventricular Overloading During Induced Anoxia with the Use of Double Lumen Catheter
}

\author{
Akira Mito \\ The Department of Medicine, Osaka Medical Colledge, Takatsuki \\ (Director Prof. K. Hara)
}

(Received for Publication, August 31, 1962)

$\mathrm{S}^{\mathrm{r}}$ UDIES ON CARDIOVASCULAR hemodynamics were almost nonexistent before intravenous catheterization was introduced by Forssman. Now that intravenous catheterization is used widely, studies on pulmonary function have made tremendous advances, whereas that concerning the right heart seems to be lagging. behind. Studies on cardiac work and metabolism are directed mostly towards the left ventricle. This is in part due to the difficulty in evaluating the right ventricular changes on the electrocardiogram and problems concerning; loads on the right ventricle have been investigated only in relation to studies on pulmonary function.

The fact that right heart failure can occur more easily than left heart failure during anoxia, can be understood from the studies by Peters \& Roos $^{1)}$, Fishmann ${ }^{2)}$, Doyle ${ }^{\text {?) }}$, Aviado ${ }^{4)}$, and Cournand ${ }^{5)}$. That is, the pulmonary flow may increase or decrease and its change is not uniform, but the pulmonary vascular bed contracts and increases the pulmonary vascular resistance, causing pulmonary hypertension. When the works of the right and left sides of the heart during anoxia are compared, studied done in our department show that $10 \% \mathrm{O}_{2}$ for 20 minutes does not always cause an increase of systemic blood pressure, but the pulmonary blood pressure was always elevated. As for the work of the each ventricle, that of the left ventricle decreases while the right increases markedly ${ }^{(i)}$. (Fig. 1) This increase of right ventricular work corresponds to the "pressure overloading" mentioned in Cabrera's

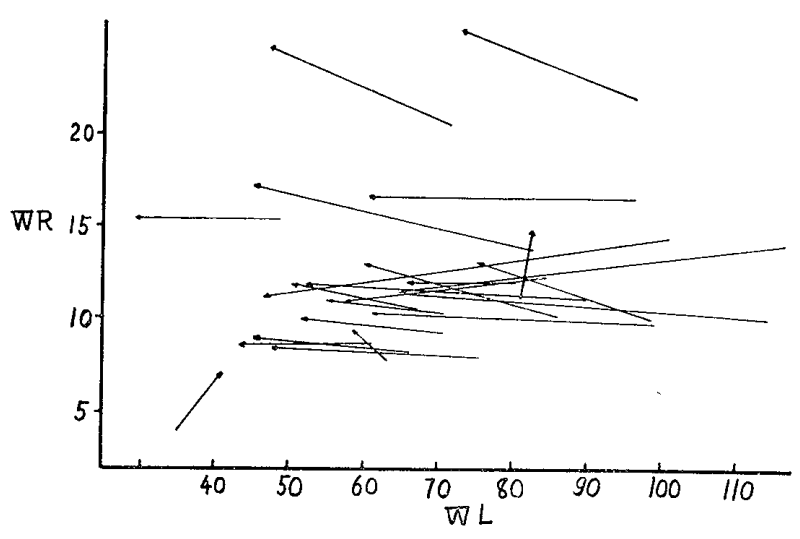

Fig. 1. Correlation of the right and left cardiac work during induced anoxia. (Matsushima, Mito)

paper and is due to pulmonary hypertension ${ }^{\text {i) }}$. This point was studied by using a double lumen catheter.

The two holes at the point of the double lumen catheter open in different angles to the blood flow. Therefore, as a preliminary experiment, the pressure recorded by the electromanometer with the catheter in flowing water was studied. It was confirmed that at flows of three to six liters, the pressure recorded through both lumens were identical and that it could be used for human catheterization.

Case materials consisted of 13 cases with no disturbances in the blood flow of the right heart system. The point of the double lumen catheter was placed in the trunk of the pulmonary artery and the side hole in the right ventricle and simultaneous recordes of pressures, electrocardiogram, phonocardiogram and respiration curves were obtained.

If the side hole of the double lumen 


\section{A}

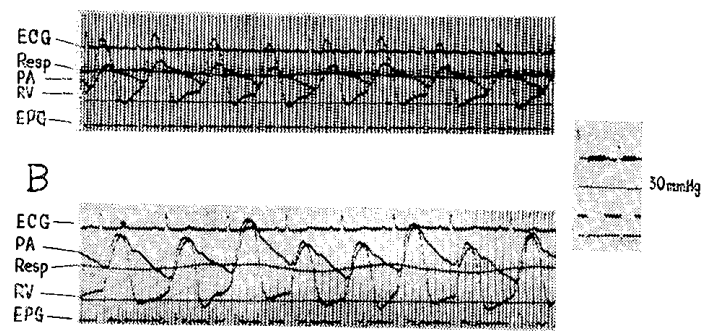

Fig. 2. Simultaneous recordes of right veutricular and pulmonary pressure, electrocardiogram, phonocardiogram and respiration curves.

A) During open air breathing.

B) During $10 \% \mathrm{O}_{2}$ breathing for 20 minutes on the same examines.

catheter is placed immediately below the pulmonic valve, i. e. If the out flow of the right ventricle is recorded, the systolic pressures of the right ventricle and pulmonary artery are superimposed. Also, if the side hole is pulled back to the tricuspid valve, the hole is clossed by the valve in systole, causing changes in the recorded curve. Therefore, the study was done only on cases where the side hole was placed at the middle of the right ventricle and where the second heart sound and intersecting point of the both pressure curve coincided. As for the measurement of time, in order to correct the difference in the cardiac rhythm, all $\mathrm{R}-\mathrm{R}$ times on the electrocardiogram were considered as 100 and the time from the $Q$ to the intersecting points of the pressure curves and the maximum amplitude on the phonocardiogram were expressed in percentage.

The first intersecting point of the pulmonary and right ventricular curves is the point at which the pulmonic valve opens and blood is expelled in to the pulmonary artery and the latter intersecting point is where the valve closes. Therefore, the relation between these points and the phonocardiogram was studied.

It is said that the first heart sound is due to closing of atrioventricular valves, muscular vibration etc. When this relationship is investigated, the first maximum amplitude of the first heart sound sometimes coincide with the closure of the tricuspid valve, but usually is more delayed and is seen before the pulmonic valve opens.
The second sound is distributed over a wide area, perhaps because it is far from the $Q$, but the latter intersecting point of the pressure curves also are distributed in the same fashion and the two agree well. Therefore, if the time laps from the second sound to the intersecting point is considered, the sccond sound agrees well is the point. In other words, the first sound and the closing of the valve dose not always coincide and this can be considered as being due to factors such as the blood stream murmur and myocardial vibration sound which Luisada indicated besides the sound due to closure of the mitral valve. At the time of the second sound, when the blood flow is also stopped, the intersecting point of both pressure curves coincide well with the sound. Therefore, the second sound can be considered to be the sound caused by the closing valves. (Fig. 3)

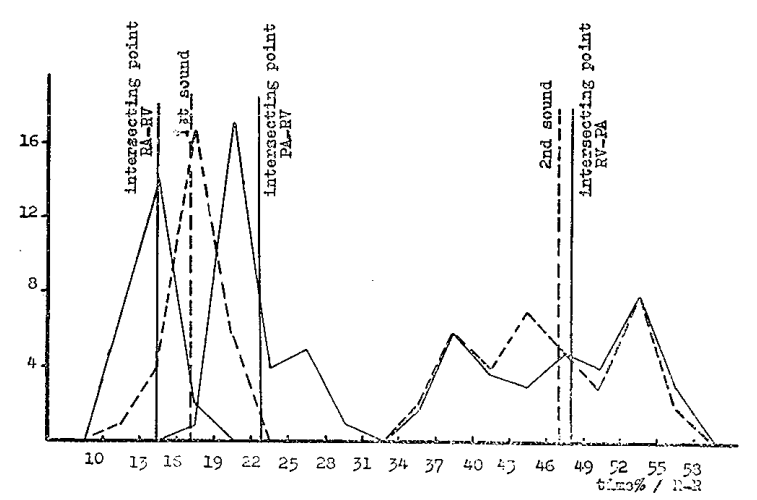

Fig. 3. Correlation of the heart sound and intersecting points of the pressure curves.

The fact that respiration influences the intracardiac pressure has been reported by Richardes, Fishman and others ${ }^{s)}$. This was studied in several cases. The pulse rate increases during inspiration and decreases during exspiration, but contrary to this, the peaks of the pulmonary artery and right ventricle pressure curves are delayed during inspiration and quickened during exspiration. The pressure drops during inspiration and rises during exspiration. These findings agree with that of Kilburn $^{97}$ who noted that the pulmonary vascular resistance increases during positive pressure breathing.

The systolic pulmonary artery pressure and systolic right ventricular pressure rise 
parallel to each other. In cases where there are no valvular diseases, the intersecting point of these two curves indicate the opening and closing points of the pulmonic valves. Therefore, if one considers the time between the opening and closing points of the valve as the "effective arterial expulsion time", no relation between this time and the mean right ventricular pressure was found, but the time was shorter as the mean pulmonary artery pressure was higher. (Fig. 4)

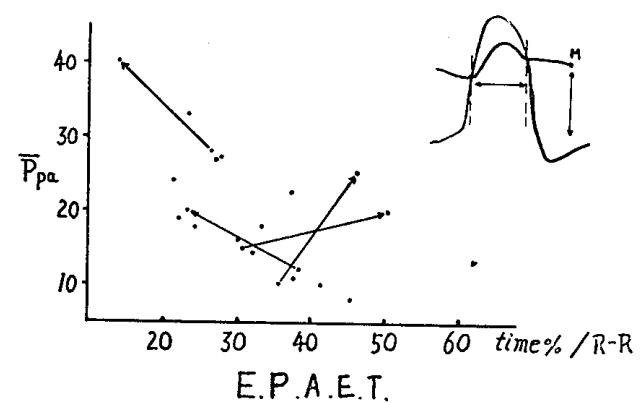

Fig. 4. Relationship between the pulmonary arterial pressure and the effective arterial expulation time.

Also, the relation between the mean pulmonary artery pressure and the pressure difference between the opening and closing points became larger as the mean pulmonary artery pressure rose. (Fig. 5)

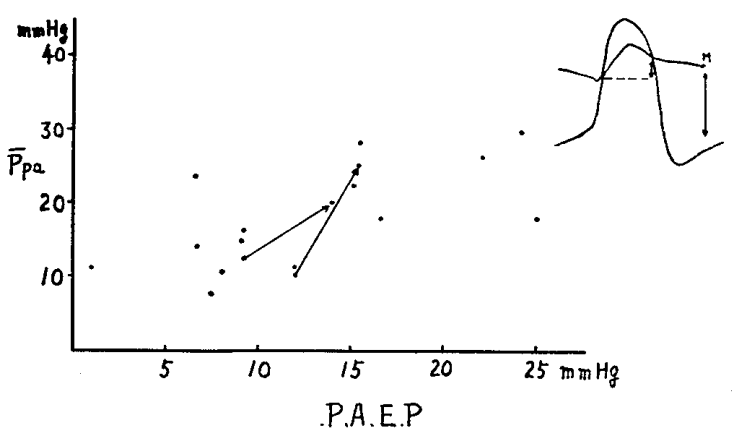

Fig. 5. Relationship between the mean pulmonary arterial pressure and the pressure difference between the opening and the closing points. (pulmonary artery expulsion psessure).

Next, in order to consider what the area outlined by the right ventricle and pulmonary artery pressures mean, the pressure difference between the systolic right ventricular and pulmonary artery pressures was used instead of the area at this time and this was compared with the mean pulmonary artery pressure. It was found that this pressure difference was smaller as the mean pulmonary artery pressure was higher. The opposite result was noted in right ventricular load due to pulmonary stenosis, where the pressure difference increase due to the stenosis and the cardiac efficiency, becomes poor. However, in pulmonary hypertension without stenosis, this pressure difference is small and the pressure in the right ventricle is transmitted well to the pulmonary artery. (Fig. 6)

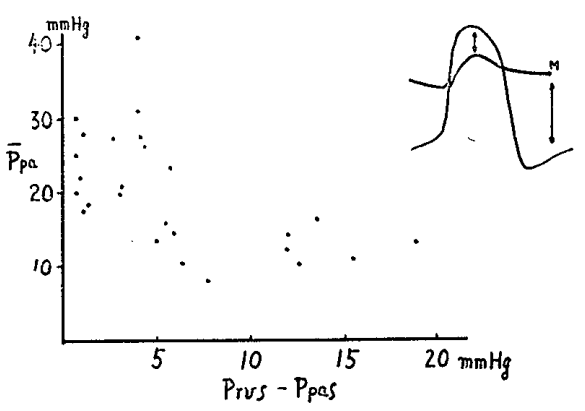

Fig. 6. Relationship between the mean pulmonary artery pressure and the pressure difference of the systoric right ventricular and pulmonary artery pressure.

\section{SUMMARY}

A double lumen catheter was employed to examine the hemodynamics of the right heart. Also, the factors which cause pulmonary hypertension in anoxia were determined.

The relation between the heart sound and the intersecting point of the ventricular and arterial pressure curves were that the second sound and the closing point of the pressure curve coincide well, but the first sound did not coincide well with the opening point of the curve probably because interference of sounds due to blood flow, myocardial vibration etc.

When the pulmonary artery pressure rises (this includes the condition during induced anoxia) the effective expulsion time of the right ventricle shortens and the ventricle is forced to expell a certain amount of blood within a short time.

In cases with no obstruction in blood flow, the pressure difference between the systolic right ventricular and pulmonary pressures decreases as the mean pulmonary arterial pressure rises. Thus, in combination with the fact noted in the above section, the right 
ventricle is placed in a state of an increased pressure load. When this state becomes more severe, the possible occurrence of right ventricular insufficiency can be predicted.

This paper was made possible with the cooperation of Drs. Shinji Watanabe, H. YoshiNAKa, K. YazU, R. Yazima, K. Nariyama, K. Asakuma, I. Suemitsu, Shigeki Watanabe and T. OKuI.

The auther wishes to express sincere gratitude to Prof. K. Hara for his guidance.

\section{REFERENCE}

1) Peters, R. M., Roos, A., Am. J. Physiol. 171: 250,
1962

2) Fishman, A. P., Fritts, H. W. Jr., Cournand, A., Circulation, 22: 204, 1960.

3) Doyle, J. T., Wilson, J. S., Warren, J. V., Circulation 5: 263, 1952.

4) Aviado, D. M. Jr., Cerletti, A. and other, Am. J. Physiol, 169: 460, 1952.

5) Coulnand, A., Circulation, 2: 641, 1950.

6) Matsushima, T., Japan Circulation Jour., 24: 1052 1960.

7) Cabrera, E., Caxiola, A., Am. Heart J., 60: 296, 1960.

8) Richards, D. W., Fishman, A. P., Pulmonary Emphysema (Barch, A. L., Bickman, H. H.), Williams \& Wilkins Co. 1956.

9) Kilburn, K. H., Sieker, H. O., Circul. Reserch, 8: $660,1960$. 